Editorial

Herz 2019 • 44:674-675

https://doi.org/10.1007/s00059-019-04867-1

(c) Springer Medizin Verlag GmbH, ein Teil von Springer Nature 2019

\author{
R. Dörr' ${ }^{1}$ B. Maisch ${ }^{2,3}$ \\ 'Praxisklinik Herz und Gefäße, Dresden, Deutschland \\ ${ }^{2}$ Herz- und Gefäßzentrum Marburg, Marburg, Deutschland \\ ${ }^{3}$ FB Medizin, Philipps-Universität Marburg, Marburg, Deutschland
}

\title{
ESC-Leitlinien 2019: seit 25 Jahren Konsens in Europa
}

Liebe Leser,

während der politische Konsens in Europa zunehmend schwieriger zu werden scheint, zeigen die wissenschaftlichen Leitlinien der Europäischen Gesellschaft für Kardiologie (ESC), die in diesem Jahr ihr 25-jähriges Leitlinienjubiläum feiern, alljährlich in beispielhafter Weise, dass ein Konsens in Europa sinnvoll und problemlos möglich ist. Beim Jahreskongress der ESC 2019 in Paris wurden vor diesem Hintergrund wieder 5 neue Leitlinien zu den Themenkreisen „Chronic Coronary Syndromes“ [1], „Diabetes, Pre-Diabetes and Cardiovascular Diseases" [2], „Dyslipidaemias“ [3] „Acute Pulmonary Embolism“ [4] und „Supraventricular Tachycardia“ [5] vorgestellt.

Ein prinzipielles Problem dieser englischsprachigen ESC-Leitlinien besteht jedoch darin, dass sie mit einem Umfang von bis zu 90 Seiten im Volltextformat und bis zu 50 Seiten in der Pocket-Version nicht immer leicht lesbar sind. Aus diesem Grund wurde vor 10 Jahren bei der Herausgebersitzung der Zeitschrift Herz anlässlich der Frühjahrstagung 2009 der Deutschen Gesellschaft für Kardiologie (DGK) in Mannheim die Idee aufgegriffen, die alljährlich erscheinenden, umfangreichen Leitlinien der ESC in einem eigenen Leitlinienheft der Zeitschrift Herz von deutschsprachigen Koautoren inhaltlich komprimiert und thematisch fokussiert kommentieren $\mathrm{zu}$ lassen. Die zentralen Fragestellungen, die dabei beantwortet werden sollten, waren: Was ist neu, und was ist besonders wichtig? Ziel sollte es sein, mit diesem Leitlinienheft die Verbreitung der Kernbotschaften der relativ komplexen ESC-
Leitlinien im deutschen Sprachraum zu beschleunigen. Das erste Leitlinienheft der Zeitschrift Herz erschien daraufhin als Heft Nr. 8 im Dezember 2010. Die weitere Entwicklung war dann fast ein Selbstläufer, im Dezember 2019 erscheint jetzt das vorliegende Leitlinienheft Nr. 8/2019 der Zeitschrift Herz bereits zum zehnten Mal - auch dies schon ein kleines Jubiläum.

Im aktuellen Heft werden die neuen ESC-Leitlinien zu den chronischen Koronarsyndromen [1] von S. Silber, München kommentiert. Wesentliche Neuerungen im Vergleich zu den älteren Leitlinien zur stabilen koronaren Herzkrankheit (KHK) von 2013 sind der Begriff des chronischen Koronarsyndroms, der an die Stelle der stabilen KHK getreten ist, eine erhebliche Absenkung der KHK-Vortestwahrscheinlichkeiten im Vergleich zu 2013 und eine noch weitere Herabstufung des konventionellen Belastungs-EKG auf die Empfehlungsklasse IIb und den Evidenzgrad B. Das Belastungs-EKG, das nur eine Sensitivität von $58 \%$ und eine Spezifität von $62 \%$ bei der Erkennung von Stenosen größer als $50 \%$ hat, ist demnach für ein sicheres „rule-out“ oder „rule-in“ einer stenosierenden KHK in der Mehrzahl aller Fälle diagnostisch ungeeignet. Statt des Belastungs-EKG werden funktionelle (Stressecho, SPECT [„,single photon emission computed tomography"] oder Stress-MRT [Magnetresonanztomographie]) oder anatomische bildgebende Verfahren (Kardio-CT) mit Empfehlungsklasse I und Evidenzgrad B an den Anfang der diagnostischen Hierarchie gestellt. Bis sich diese Vorstellung in kardiologischen und internistischen Praxen etabliert hat, dürfte es noch eine Zeit lang dauern.

Die neuen ESC/EASD(European Association for the Study of Diabetes)-Leitlinien zu Diabetes, Prädiabetes und kardiovaskulären Erkrankungen [2], die die Vorgängerversion ebenfalls aus dem Jahr 2013 ersetzen, werden von N. Marx, Aachen zusammengefasst. In die Empfehlungen dieser Leitlinien sind die in den letzten Jahren veröffentlichten Daten aus großen kardiovaskulären Endpunktstudien mit neuen antidiabetisch wirksamen Substanzen eingeflossen und haben $\mathrm{zu}$ einer völlig neuen Positionierung von blutzuckersenkenden Medikamenten in der Reduktion kardiovaskulärer Ereignisse bei Patienten mit Diabetes mellitus geführt.

Die neuen europäischen Leitlinien zu Dyslipidämien 2019 [3], die jetzt schon nach 3 Jahren die Vorgängerversion von 2016 ablösen, werden von J. L. Katzmann, A. Tünnemann-Tarr und U. Laufs aus Leipzig bearbeitet. Ergebnisse großer randomisierter Studien, insbesondere mit den PCSK9(Proproteinkonvertase Subtilisin/Kexin Typ 9)-Inhibitoren, die seit der Veröffentlichung der letzten Leitlinienversion im Jahr 2016 publiziert wurden, konnten eine Reduktion kardiovaskulärer Ereignisse durch eine Senkung des LDL(„low-density lipoprotein“)-Cholesterins über die alten Zielwerte hinaus zeigen. Der Fokus der Empfehlungen liegt deshalb auf einer möglichst frühen und möglichst großen absoluten LDL-Cholesterin-Senkung. Aufgrund dieser Erkenntnisse und der erweiterten medikamentösen Therapiemöglichkeiten wurden die Zielwerte für LDL-Cholesterin insbesondere für 
Patienten mit sehr hohem kardiovaskulären Risiko (LDL-Cholesterin-Zielwert jetzt $<1,4 \mathrm{mmol} / \mathrm{l}$ [55 mg/dl]) oder mit rezidivierenden kardiovaskulären Ereignissen trotz laufender Statintherapie (LDL-Cholesterin-Zielwert jetzt $<1,0 \mathrm{mmol} / \mathrm{l}$ [40 mg/dl]) nach unten angepasst.

Die ESC-Leitlinien 2019 „Diagnostik und Management der akuten Lungenembolie“ [4], die die ältere Leitlinienversion von 2014 ersetzen, werden von R. Osteresch, A. Fach, R. Hambrecht und $\mathrm{H}$. Wienbergen aus Bremen interpretiert. Der aktuelle risikoadjustierte Algorithmus zum Management der akuten Lungenembolie bezieht den klinischen Schweregrad, die Begleiterkrankungen und eine rechtsventrikuläre Dysfunktion ein. Während bei Niedrigrisikopatienten eine frühe Entlassung und ambulante Therapie möglich sind, müssen bei Hochrisikopatienten in Abhängigkeit von der hämodynamischen Situation und den individuellen Kontraindikationen eine Reperfusionstherapie und eine hämodynamische Unterstützung erwogen werden. Alle Patienten mit akuter Lungenembolie sollten für mindestens 3 Monate therapeutisch antikoaguliert werden.

K.-H. Kuck, Hamburg kommentiert die neuen ESC-Leitlinien zum Management von Patienten mit supraventrikulären Tachykardien [5]. Bei diesen Leitlinien ist der zeitliche Abstand $\mathrm{zu}$ den alten Leitlinien aus dem Jahr 2003 mit 16 Jahren am größten. Aus diesem Grund ergeben sich teilweise gravierende Unterschiede bei den medikamentösen Therapieempfehlungen. Medikamente, die 2003 noch mit höheren Empfehlungsklassen gelistet wurden, finden sich in den neuen Leitlinien von 2019 nicht mehr wieder oder haben wie bei Sotalol wegen proarrhythmogener Effekte und erhöhter Mortalität sogar die Empfehlungsklasse III und den Evidenzgrad C bekommen. Des Weiteren haben sich die Techniken und Indikationen der Katheterablation von supraventrikulären Tachykardien in diesem langen Zeitraum ebenfalls erheblich verändert.

Die Herausgeber dieses Leitlinienhefts 8/2019 wünschen den Lesern der Zeitschrift Herz erneut viel Vergnügen bei der Lektüre dieses Hefts, verbunden mit den besten Wünschen für den politischen Konsens in Europa, ein frohes Weihnachtsfest 2019 und ein gesundes und friedvolles neues Jahr 2020.

Ihre

Dr. med. Rolf Dörr

Prof. Dr. med. Bernhard Maisch

\section{Korrespondenzadresse}

\section{Dr. med. R. Dörr}

Praxisklinik Herz und Gefäße

Heinrich-Cotta-Str. 12, 01324 Dresden,

Deutschland

doerr@praxisklinik-dresden.de

Interessenkonflikt. R. Dörr und B. Maisch geben an, dass kein Interessenkonflikt besteht.

\section{Literatur}

1. Knuuti J, Wijns W, Saraste A et al (2019) 2019 ESC guidelines for the diagnosis and management of chronic coronary syndromes. Eur Heart J. https:// doi.org/10.1093/eurheartj/ehz425

2. Cosentino F, Grant PJ, Aboyans V et al (2019) ESC guidelines on diabetes, pre-diabetes, and cardiovascular diseases developed in collaboration with the EASD. Eur Heart J. https://doi.org/10. 1093/eurheartj/ehz486

3. MachF, BaigentC, Catapano ALetal (2019)ESC/EAS guidelines for the management of dyslipidaemias: lipid modification to reduce cardiovascular risk. Eur Heart J.https://doi.org/10.1093/eurheartj/ehz455

4. Konstantinides SV, Meyer G, Becattini Cet al (2019) ESC guidelines for the diagnosis and management of acute pulmonary embolism developed in collaboration with the European Respiratory Society (ERS). Eur Heart J. https://doi.org/10.1093/ eurheartj/ehz405

5. Brugada J, Katritsis DG, Arbelo E et al (2019) ESC Guidelines for the management of patients with supraventricular tachycardia. Eur Heart J. https:// doi.org/10.1093/eurheartj/ehz467
Kinderschutz in der Medizin: Online-Grundkurs für alle Gesundheitsberufe

CME-zertifiziertes eLearning-Modul, Registrierung noch bis 19.03 .2020 kostenfrei möglich

Gesundheitsfachkräfte sind die ersten Ansprechpartner für Betroffene von Kindesmisshandlungen. Häufig wird Misshandlung in Kliniken und Praxen jedoch nicht erkannt und es besteht Unsicherheit über die Handlungsmöglichkeiten. Deswegen ist die effektive und nachhaltige Weiterbildung von Gesundheitsfachkräften so bedeutsam.

Im Rahmen eines vom Bundesministerium für Gesundheit geförderten Projektes wird der Online-Kurs "Kinderschutz in der Medizin - ein Grundkurs für alle Gesundheitsberufe" an der Klinik für Kinder- und Jugendpsychiatrie / Psychotherapie des Uniklinikums Ulm entwickelt und evaluiert (https://grundkurs.elearning-kinderschutz.de/). Der Kurs richtet sich an alle Angehörigen der Gesundheitsberufe. Er bietet einen Überblick über die aktuelle Rechtslage, die Epidemiologie und Diagnostik der Misshandlungsformen und Vorgehen und Leitlinien im Kinderschutz. Neben der Wissensvermittlung wird besonderer Wert auf fallbasiertes Lernen gelegt. In einem Praxisbereich werden Fallbeispiele aus verschiedenen medizinischen Bereichen bearbeitet.

Das Kursprogramm wird regelmäßig evaluiert, um die Lernplattform und die Lerninhalte kontinuierlich weiterzuentwickeln. Bei allen Berufsgruppen zeigt sich eine signifikante Zunahme an Wissen und Kompetenzen über die Dauer des Kurses hinaus. Die Inhalte des Kurses werden als relevant für den medizinischen Arbeitsalltag beurteilt. Fast alle Absolvent_innen des Kurses würden den Kurs an Kolleg_innen weiterempfehlen.

Die Bearbeitungszeit beträgt ca. 30 Stunden und kann im Zeitraum von einem halben Jahr frei eingeteilt werden.

Die Anmeldung zum Kurs ist kostenlos und noch bis 19.03.2020 unter https://grundkurs.elearning-kinderschutz.de/registrierung möglich. 